\title{
Surgery for upper gastrointestinal tract
}

Incidence of gastric cancer is mainly in East Asia, and minimally invasive surgery such as endoscopic mucosal dissection, laparoscopic surgery and robotic surgery for gastric cancer has been developed mainly in this area. On the other hand, from the viewpoint of minimally invasive surgery for stomach diseases, various surgical techniques including benign diseases are performed in the world. In recent years, new techniques based on new concepts such as metabolic surgery for morbid obesity and combined operations of endoscope and laparoscope for submucosal tumor are being developed.

In this way, the minimally invasive surgical technique surrounding the stomach disease continues to progress steadily. We can make further progress by learning new techniques from other fields without sticking to our own clinical working.

This time, not only the ingenuity in laparoscopic surgery for gastric cancer, various procedures such as minimally invasive surgery for submucosal tumor typified by GIST, metabolic surgery for morbid obesity, etc., are drafted from experts in various fields in the world. Although in different areas, I edited this volume, hoping that each technique fuses or influences each other and newer concepts and techniques will be developed. I also hope that all articles will be useful for clinical practice in all readers from tomorrow.

\section{Acknowledgements}

None.

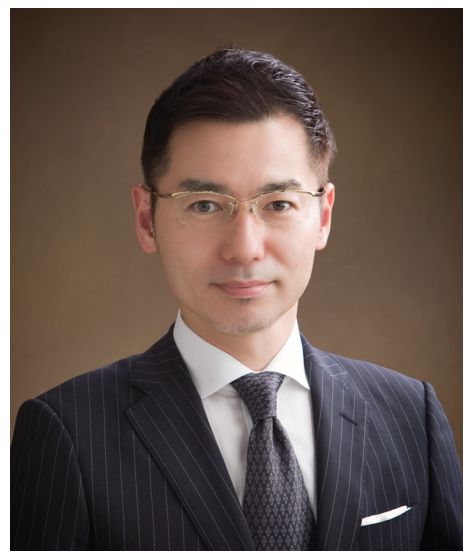

Noriyuki Inaki

Noriyuki Inaki, $\mathrm{MD}, \mathbf{P h D}$

Department of Gastroenterological Surgery, Ishikawa Prefectural Central Hospital, Kanazawa, Japan.

(Email:n.inaki@viola.ocn.ne.jp)

doi: $10.21037 / \operatorname{tgh} .2017 .11 .04$

Conflicts of Interest: The author has no conflicts of interest to declare.

View this article at: http://dx.doi.org/10.21037/tgh.2017.11.04

doi: $10.21037 / \operatorname{tgh} .2017 .11 .04$

Cite this article as: Inaki N. Surgery for upper gastrointestinal tract. Transl Gastroenterol Hepatol 2017;2:90. 